\title{
Correction to: Evaluation and ranking of different gridded precipitation datasets for Satluj River basin using compromise programming and f-TOPSIS
}

\author{
Bratati Chowdhury ${ }^{1,2} \cdot$ N. K. Goel ${ }^{1} \cdot$ M. Arora $^{3}$ \\ Published online: 12 November 2020 \\ (C) Springer-Verlag GmbH Austria, part of Springer Nature 2020
}

\section{Correction to: Theoretical and Applied Climatology https://doi.org/10.1007/s00704-020-03405-y}

The original version of this article unfortunately contained mistakes. The proof corrections in Eq. 9 and Tables 3 and 5 were unfortunately not implemented. The corrected data are given below. In addition, affiliations of the 1st author have been updated. The original article has been corrected.

$$
C=\frac{\text { DS_Minus }}{\text { DS_Minus + DS_Plus }}
$$

The online version of the original article can be found at https://doi.org/ 10.1007/s00704-020-03405-y

Bratati Chowdhury

bchowdhury@hy.iitr.ac.in

1 Department of Hydrology, Indian Institute of Technology Roorkee, Roorkee, Uttarakhand 247667, India

2 Faculty of Technology, Uttar Banga Krishi Viswavidyalaya, Cooch Behar, West Bengal 736 165, India

3 National Institute of Hydrology Roorkee,

Roorkee, Uttarakhand 247667, India 
Table 3 Rank of the different gridded precipitation dataset for each station from Compromise Programming along with its parameter (Lp) and performance indicator payoff matrix (RMSE, CC, and SS)

\begin{tabular}{|c|c|c|c|c|c|c|}
\hline & \multicolumn{6}{|c|}{ Root Mean Square Error (RMSE) } \\
\hline & CFSR & MERRA & APHRODITE & $\begin{array}{l}\text { ERA- } \\
\text { Interim }\end{array}$ & IMD & PFD \\
\hline Bhakra & 0.81 & 0.78 & 0.56 & 0.79 & 0.59 & 1.00 \\
\hline Berthin & 0.75 & 0.73 & 0.50 & 0.72 & 0.54 & 1.00 \\
\hline Daslehra & 0.79 & 0.76 & 0.54 & 0.77 & 0.58 & 1.00 \\
\hline Kahu & 0.80 & 0.77 & 0.55 & 0.77 & 0.62 & 1.00 \\
\hline Kasol & 0.78 & 0.75 & 0.54 & 0.73 & 0.53 & 1.00 \\
\hline Kuddi & 0.80 & 0.78 & 0.56 & 0.77 & 0.59 & 1.00 \\
\hline Rampur & 0.67 & 0.61 & 0.49 & 0.57 & 0.46 & 1.00 \\
\hline \multirow[t]{2}{*}{ Suni } & 0.72 & 0.69 & 0.44 & 0.67 & 0.49 & 1.00 \\
\hline & \multicolumn{6}{|c|}{ Correlation Coefficient (CC) } \\
\hline Bhakra & 0.01 & 0.07 & 0.99 & 0.46 & 0.91 & 0.14 \\
\hline Berthin & 0.00 & 0.05 & 0.99 & 0.44 & 0.93 & 0.15 \\
\hline Daslehra & 0.01 & 0.06 & 0.99 & 0.44 & 0.92 & 0.18 \\
\hline Kahu & 0.02 & 0.04 & 0.99 & 0.40 & 0.82 & 0.14 \\
\hline Kasol & 0.02 & 0.02 & 0.97 & 0.46 & 0.94 & 0.15 \\
\hline Kuddi & 0.02 & 0.04 & 0.99 & 0.44 & 0.91 & 0.14 \\
\hline Rampur & 0.01 & 0.01 & 0.98 & 0.40 & 0.94 & 0.09 \\
\hline \multirow[t]{2}{*}{ Suni } & 0.01 & 0.02 & 0.99 & 0.38 & 0.91 & 0.12 \\
\hline & \multicolumn{6}{|c|}{ Skill Score (SS) } \\
\hline Bhakra & 0.94 & 0.97 & 0.97 & 0.88 & 0.99 & 0.90 \\
\hline Berthin & 0.94 & 0.97 & 0.98 & 0.88 & 0.98 & 0.90 \\
\hline Daslehra & 0.94 & 0.98 & 0.97 & 0.88 & 0.94 & 0.90 \\
\hline Kahu & 0.92 & 0.96 & 0.94 & 0.86 & 0.95 & 0.97 \\
\hline Kasol & 0.94 & 0.91 & 0.97 & 0.87 & 0.98 & 0.97 \\
\hline Rampur & 0.92 & 0.91 & 0.91 & 0.84 & 0.93 & 0.96 \\
\hline \multirow[t]{2}{*}{ Suni } & 0.93 & 0.97 & 0.90 & 0.85 & 0.92 & 0.90 \\
\hline & \multicolumn{6}{|c|}{ Lp Matric } \\
\hline Bhakra & 0.34626 & 0.32585 & 0.00002 & 0.18894 & 0.03201 & 0.30111 \\
\hline Berthin & 0.34206 & 0.32785 & 0.00001 & 0.19103 & 0.02503 & 0.29246 \\
\hline Daslehra & 0.34210 & 0.32620 & 0.00002 & 0.19228 & 0.02746 & 0.28163 \\
\hline Kahu & 0.36889 & 0.36036 & 0.00006 & 0.22721 & 0.06788 & 0.32355 \\
\hline Kasol & 0.33194 & 0.33289 & 0.00884 & 0.18353 & 0.00001 & 0.28736 \\
\hline Kuddi & 0.34992 & 0.34309 & 0.00002 & 0.20180 & 0.03232 & 0.30698 \\
\hline Rampur & 0.33652 & 0.33733 & 0.00825 & 0.20445 & 0.00007 & 0.30935 \\
\hline \multirow[t]{2}{*}{ Suni } & 0.34620 & 0.34051 & 0.00011 & 0.21740 & 0.02980 & 0.30646 \\
\hline & \multicolumn{6}{|c|}{ Rank from Compromise Programming } \\
\hline Bhakra & 6 & 5 & 1 & 3 & 2 & 4 \\
\hline Berthin & 6 & 5 & 1 & 3 & 2 & 4 \\
\hline Daslehra & 6 & 5 & 1 & 3 & 2 & 4 \\
\hline Kahu & 6 & 5 & 1 & 3 & 2 & 4 \\
\hline Kasol & 5 & 6 & 2 & 3 & 1 & 4 \\
\hline Kuddi & 6 & 5 & 1 & 3 & 2 & 4 \\
\hline Rampur & 5 & 6 & 2 & 3 & 1 & 4 \\
\hline Suni & 6 & 5 & 1 & 3 & 2 & 4 \\
\hline
\end{tabular}


Table 5 Rank of the different gridded precipitation dataset for each station from f-TOPSIS along with its parameters (DS_Plus, DS_Minus, and C)

\begin{tabular}{|c|c|c|c|c|c|c|}
\hline & \multicolumn{6}{|c|}{ Positive Ideal value (DS_Plus) } \\
\hline & CFSR & MERRA & APHRODITE & $\begin{array}{l}\text { ERA- } \\
\text { interim }\end{array}$ & IMD & PFD \\
\hline Bhakra & 3.0879 & 3.0387 & 2.2420 & 2.6668 & 2.2998 & 3.0456 \\
\hline Berthin & 3.0530 & 3.0583 & 2.2484 & 2.7372 & 2.3056 & 3.0062 \\
\hline Daslehra & 3.0690 & 3.0212 & 2.2445 & 2.7461 & 2.3028 & 2.9339 \\
\hline Kahu & 3.0599 & 3.0654 & 2.1972 & 2.7251 & 2.3553 & 3.0125 \\
\hline Kasol & 3.0676 & 3.0720 & 2.2844 & 2.6768 & 2.2844 & 2.9404 \\
\hline Kuddi & 3.0700 & 3.0754 & 2.2324 & 2.7437 & 2.2907 & 3.0260 \\
\hline Rampur & 3.0174 & 3.0261 & 2.3028 & 2.7330 & 2.3028 & 2.9647 \\
\hline \multirow[t]{2}{*}{ Suni } & 3.0237 & 3.0291 & 2.2779 & 2.7221 & 2.3292 & 2.9711 \\
\hline & \multicolumn{6}{|c|}{ Negative Ideal value (DS_Minus) } \\
\hline Bhakra & 0.1621 & 0.2290 & 0.9905 & 0.5650 & 0.9261 & 0.2359 \\
\hline Berthin & 0.1243 & 0.1296 & 0.9223 & 0.4233 & 0.8609 & 0.1888 \\
\hline Daslehra & 0.1415 & 0.2072 & 0.9571 & 0.4479 & 0.8951 & 0.2852 \\
\hline Kahu & 0.1353 & 0.1408 & 0.9725 & 0.4532 & 0.8221 & 0.2058 \\
\hline Kasol & 0.1385 & 0.1428 & 0.9050 & 0.5097 & 0.9050 & 0.2760 \\
\hline Kuddi & 0.1438 & 0.1492 & 0.9638 & 0.4537 & 0.9003 & 0.2149 \\
\hline Rampur & 0.1332 & 0.1347 & 0.8300 & 0.3892 & 0.8300 & 0.2007 \\
\hline \multirow[t]{2}{*}{ Suni } & 0.1324 & 0.1378 & 0.8628 & 0.4037 & 0.8086 & 0.2003 \\
\hline & \multicolumn{6}{|c|}{ Closeness coefficient $(\mathrm{C})$} \\
\hline Bhakra & 0.0499 & 0.0701 & 0.3064 & 0.1748 & 0.2871 & 0.0719 \\
\hline Berthin & 0.0391 & 0.0407 & 0.2909 & 0.1339 & 0.2719 & 0.0591 \\
\hline Daslehra & 0.0441 & 0.0642 & 0.2989 & 0.1402 & 0.2799 & 0.0886 \\
\hline Kahu & 0.0424 & 0.0439 & 0.3068 & 0.1426 & 0.2587 & 0.0640 \\
\hline Kasol & 0.0432 & 0.0444 & 0.2837 & 0.1600 & 0.2837 & 0.0858 \\
\hline Kuddi & 0.0447 & 0.0463 & 0.3015 & 0.1419 & 0.2821 & 0.0663 \\
\hline Rampur & 0.0423 & 0.0426 & 0.2649 & 0.1247 & 0.2649 & 0.0634 \\
\hline \multirow[t]{2}{*}{ Suni } & 0.0420 & 0.0435 & 0.2747 & 0.1292 & 0.2577 & 0.0631 \\
\hline & \multicolumn{6}{|c|}{ Rank from f-TOPSIS } \\
\hline Bhakra & 4 & 6 & 1 & 3 & 2 & 5 \\
\hline Berthin & 6 & 5 & 1 & 3 & 2 & 4 \\
\hline Daslehra & 6 & 5 & 1 & 3 & 2 & 4 \\
\hline Kahu & 6 & 5 & 1 & 3 & 2 & 4 \\
\hline Kasol & 6 & 5 & 1 & 3 & 2 & 4 \\
\hline Kuddi & 6 & 5 & 1 & 3 & 2 & 4 \\
\hline Rampur & 6 & 5 & 1 & 3 & 2 & 4 \\
\hline Suni & 6 & 5 & 1 & 3 & 2 & 4 \\
\hline
\end{tabular}

Publisher's note Springer Nature remains neutral with regard to jurisdictional claims in published maps and institutional affiliations. 